\title{
Reflexões sobre 0 ensino de gestão em saúde no internato de medicina na Faculdade de Ciências Médicas da Universidade Estadual de Campinas - Unicamp
}

Sérgio Resende Carvalho' Gastão Wagner de Sousa Campos ${ }^{2}$ Gustavo Nunes de Oliveira ${ }^{3}$

\section{Introdução}

Neste artigo procuramos descrever e analisar a experiência de criação da disciplina "Saúde Coletiva/Gestão e Planejamento em Saúde" (SC/GP) no internato do $5^{\circ}$ ano do curso de medicina da Faculdade de Ciências Médicas (FCM/ Unicamp). Esta instituição vem, desde 2001, desenvolvendo processo de mudança curricular com objetivo de graduar médicos com sólida formação geral e capazes de prestar atenção integral e de qualidade aos usuários dos serviços de saúde.

A disciplina SC/GP teve início em novembro de 2004, quando a primeira turma de alunos da Reforma Curricular iniciou o $5^{\circ}$ ano do curso de graduação. É um módulo do internato no qual grupos de quatro a cinco alunos frequentam o estágio durante dez dias úteis, cumprindo um total de quarenta horas-aula. Durante o ano letivo passam pelo internato 24 turmas, em ciclos ininterruptos com duração de aproximadamente duas semanas (dez dias úteis).

Na maioria das vezes, a disciplina se desenvolve de tal forma que os alunos são encarregados, com apoio dos professores, de elaborarem e (ou) revisarem projetos terapêuticos singulares que tenham como objeto indivíduos ou coletivos em situação de vulnerabilidade, buscando aprimorar a intervenção das equipes de saúde locais. Cabe a estas últimas sugerirem a problemática a ser estudada pelos alunos. Durante o estágio os trabalhadores participam do processo, responsabilizando-se pela sustentabilidade e continuidade da proposta.

As principais diretrizes curriculares do curso são: a) abordagem conceitual que prioriza a interface entre a clínica e a gestão; b) incorporação das atividades de ensino ao cotidiano do gestor e dos trabalhadores das unidades básicas; c) metodologia de ensino que valoriza o aprendizado significativo (Berbel, 1995), colocando o aluno em contacto direto com os usuários, a rede e os trabalhadores de saúde.

\section{Aspectos metodológicos}

Destacamos, como objeto de análise, o processo de implementação, a partir de novembro de 2004, da disciplina SC/GP, do $5^{\circ}$ ano, internato de graduação em

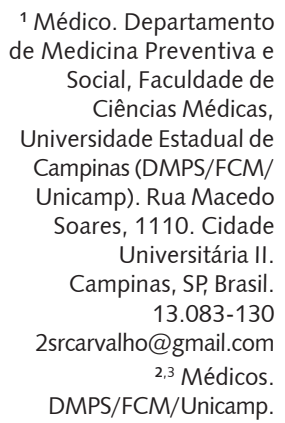

${ }^{1}$ Médico. Departamento de Medicina Preventiva e Social, Faculdade de Ciências Médicas, Universidade Estadual de Campinas (DMPS/FCM/ Unicamp). Rua Macedo Soares, 1110. Cidade Universitária II. Campinas, SP, Brasil. 13.083-130 2srcarvalho@gmail.com 2,3 Médicos. DMPS/FCM/Unicamp. 
REFLEXÕES SOBRE O ENSINO DE GESTÃO EM SAÚDE ...

medicina da FCM/Unicamp. Realizamos um estudo descritivo que abarcou os primeiros 12 meses de existência.

Trabalhamos com diferentes fontes de dados, destacando-se, a análise das respostas a questionário semiestruturado preenchido pelos alunos ao final da disciplina. Por intermédio das respostas, individuais e anônimas, os alunos avaliam a disciplina e o desempenho docente. Este questionário constitui dispositivo de monitoramento da qualidade do processo de ensino/aprendizagem por parte da coordenação docente. No processo de análise aqui realizado, valorizou-se, em especial, a reiteração de respostas com um mesmo conteúdo, buscando, no conjunto, um enunciado que as sintetizasse.

Anotações dos pesquisadores - alguns dos quais docentes da disciplina - produzidas durante o processo de preparação e realização dos estágios constituíram fonte complementar do estudo. Alguns dos processos registrados nos "diários de campo" dos pesquisadores são: acompanhamento dos alunos, reuniões com gestores e trabalhadores locais de estágio, encontros entre docentes responsáveis pela disciplina com gestores da Secretaria Municipal de Saúde de Campinas (SMS/Campinas), colegiado de docentes da disciplina, e avaliação oral por parte dos alunos da disciplina.

Utilizamos também, para esta investigação, relatos dos alunos comentando aspectos teóricos, pedagógicos e vivências na disciplina, bem como os projetos de intervenção formulados. A história do curso baseou-se, também, em documentos oficiais, entre eles: o programa e planos de ensino da disciplina em questão.

\section{O internato de gestão e planejamento}

Ao avaliar a implementação da reforma curricular do curso de medicina, iniciada em 2001, a Faculdade de Ciências Médicas da Unicamp decidiu introduzir o ensino de gestão em saúde no internato do $5^{\circ}$ ano a partir do ano de 2004.

Respondendo a esta diretiva, o Departamento de Medicina Preventiva e Social (DMPS) da FCM/ Unicamp demandou, à área de Políticas, Planejamento e Gestão, a elaboração de uma proposta. Após três meses de discussão com gestores, trabalhadores da SMS/Campinas, graduandos e pós-graduandos da FCM/Unicamp, definiu-se o programa da disciplina.

\section{Escolhas teóricas e recortes temáticos}

O primeiro desafio foi decidir as temáticas centrais do internato reconhecendo, de antemão, que a "gestão em saúde" abrange múltiplos sentidos.

A opção teórico-conceitual e o recorte temático tomaram, como base, a produção teórica sobre Planejamento e Gestão construída ao longo dos anos no interior do DMPS. A partir do entendimento de que, na saúde, o trabalho joga um papel central no cotidiano institucional, a formulação conhecida nos anos 1990 como "projeto em Defesa da Vida" (DV) preconiza que o tema da gestão em saúde é indissociável e orgânico ao debate sobre o cuidado e a clínica (Carvalho, 2005; Silva Jr., 1998).

Ao abordar o tema do cuidado, o DV sugere a ampliação do sentido e das práticas clínicas, propondo em lugar de técnicas semióticas tradicionais, que neutralizam a interferência do social e da subjetividade na prática clínica, uma prática holística na qual se valorize a relação intersubjetiva, uma vez que "o processo de diagnóstico e de cura é sempre uma relação entre sujeitos nunca redutível a uma objetividade positiva", sempre influenciado pela história social e pelas características psíquicas dos sujeitos envolvidos (Campos, 1992, p.184). Considera que a clínica tem muito a ser criticada, mas tem, também, muito a dizer. A clínica que se pleiteia é uma "outra clínica", que seja capaz de intervir sobre as disfunções biológicas por meio da prevenção, do diagnóstico, da cura e da reabilitação, mas também como “um processo e um espaço de produção de relações e intervenções, que se dá de modo partilhado, e no qual há um jogo entre necessidades e modos tecnológicos de agir" (Merhy, 2002, p.189).

Preconiza, por outro lado, uma gestão que tenha como objetivo ampliar a capacidade de análise e de intervenção dos sujeitos em distintos planos da vida social, propondo que se realizem, no interior das instituições, distintas funções, entre as quais: 
uma clássica, de administrar e planejar processos de trabalho objetivando a produção de valores de uso [...] outra de caráter político, a co-gestão como uma forma de alterar as relações de poder e construir a democracia em instituições [e] uma pedagógica e terapêutica [que se refere à] capacidade que os processos de gestão têm de influir sobre a constituição de sujeitos. (Campos, 2000, p.4)

A partir deste pano de fundo teórico-conceitual, buscou-se compor interesses e demandas presentes no programa de reforma curricular, em curso na faculdade, com as necessidades dos alunos e com os potenciais cenários de práticas na rede do Sistema Único de Saúde (SUS) em Campinas. A reduzida carga horária da disciplina (quarenta horas), concentrada em dez dias seguidos, e o perfil dos alunos de $5^{\circ}$ ano de graduação em Medicina com prática concentrada em ambiente hospitalar - foram, igualmente, fatores que pesaram no processo decisório.

O curso foi estruturado tendo como eixo a inseparabilidade da gestão e da prática clínica no fazer/ pensar saúde. Com isso, buscou-se mostrar aos alunos a importância de incorporar o tema na formação médica, tendo em vista as características contemporâneas do trabalho em saúde, que é marcado pelo caráter interdisciplinar da prática e pela existência de uma complexa rede de cuidado em saúde.

Qualificar a prática clínica por meio da valorização do encontro trabalhador/usuário e da gestão da clínica constitui um dos eixos da disciplina. Isto tem demandado a busca por cenários pedagógicos nos quais os alunos tenham a oportunidade de vivenciar práticas transdisciplinares com as equipes de saúde, e elaborar projetos que efetivem linhas de cuidado no interior da rede SUS e junto a equipamentos e arranjos sociais extrassetor saúde (Cecílio, 2001; Merhy, 1998).

Do encontro entre o recorte teórico acima mencionado e as problemáticas e projetos dos centros de saúde que serviriam de campo de estágio, elaborou-se um conjunto de eixos temáticos que deveriam referenciar as atividades dos estagiários, a saber: clínica ampliada; projeto terapêutico singular; trabalho em equipe, equipes de referência e de apoio matricial, interdisciplinaridade, gestão da atenção em saúde, gestão colegiada e rede de cuidados.

Os temas acima são discutidos com base em problemas de gestão ou de atenção à saúde, no cotidiano dos serviços. A ênfase na eleição e na abordagem dos casos varia de acordo com a demanda das equipes de trabalhadores e (ou) dos gestores e do processo de investigação/intervenção em si desenvolvido pelos alunos com apoio dos docentes, dos trabalhadores e dos gestores. A partir de realidades concretas são disparados processos de reflexão que têm, como subsídio, a vivência prática e textos teóricos de apoio.

\section{Proposta pedagógica}

O estágio tem como referência centros de saúde da SMS/Campinas. A escolha dos mesmos levou em conta a adesão à proposta da disciplina por parte das equipes locais. Foram valorizadas, igualmente, as unidades que vinham vivenciando processos internos de mudança sob a influência de diretrizes do projeto tecnoassistencial do DV (Campinas, 2001; Carvalho, Campos, 2000).

Ao longo do ano, ocorre um revezamento entre os serviços que são campos de estágio, de modo a garantir um intervalo ( 15 a 45 dias, de acordo com o número de unidades envolvidas) sem a presença de alunos. Desta forma, procura-se atenuar o eventual desgaste dos trabalhadores da unidade advindo do esforço extra que se faz para receber alunos e docentes da Unicamp.

Com base na demanda de distintas equipes de referência de um dos centros de saúde, os alunos investigam uma situação problemática - muitas vezes um caso clínico complexo e (ou) com o qual os trabalhadores têm dificuldade de lidar - e buscam refletir e propor intervenções para a mesma. Ao final do estágio, os alunos apresentam suas propostas objetivando qualificar o trabalho das equipes. Estas propostas tomam, muitas vezes, a forma de um projeto terapêutico singular, que é discutido e validado no coletivo da equipe responsável pelo caso. Em geral, estes casos envolvem vários serviços de diferentes níveis hierárquicos e, mesmo, de outros setores. Os alunos estudam o percurso do usuário, visitando territórios no entorno do centro de saúde e nos serviços da rede SUS/Campinas, discutindo o 
REFLEXÕES SOBRE O ENSINO DE GESTÃO EM SAÚDE ...

projeto com profissionais que intervieram no caso ou que poderiam, potencialmente, contribuir para a qualificação do mesmo.

A avaliação discente procura aferir a competência (Lima, 2004) do aluno em valer-se de instrumentos de gestão para organizar intervenções clínicas ou sanitárias e estrutura-se a partir de três eixos. O primeiro é o próprio projeto terapêutico elaborado pelo coletivo de alunos e validado pela equipe e pelos usuários envolvidos. O segundo eixo é a avaliação individual da postura de cada aluno diante da equipe de saúde, do usuário, de seus familiares, assim como de sua rede social. Em algumas situações utiliza-se, como terceiro elemento avaliativo, um exercício, de caráter individual, no qual o aluno é solicitado a apresentar sugestões para a reorganização de algum serviço de saúde tendo como referência debates e textos sobre os eixos temáticos acima mencionados.

\section{Reflexões sobre o internato de gestão em saúde}

Os dados coletados atestam que a disciplina tem logrado demonstrar, à maioria dos alunos, a relevância de um arcabouço conceitual que traz a Clínica para o interior da Gestão e a Gestão para o interior da Clínica (Carvalho, Cunha, 2006). Ao elaborarem projetos terapêuticos singulares (Oliveira, 2007; Cunha, 2005), os graduandos vêm tendo a oportunidade de vivenciar e compreender a importância dos distintos fóruns de gestão do processo de trabalho, existentes nas unidades visitadas, para as práticas de saúde (p.ex.: colegiado gestor, reunião de equipes, fórum dos trabalhadores, conselho local de saúde, núcleo de Saúde Coletiva, oficinas de avaliação e de planejamento). Conseguem, igualmente, conhecer e avaliar o papel da rede de atenção secundária para a atenção em saúde.

Os alunos avaliam positivamente esse processo. Ao serem indagados sobre a pertinência dos conteúdos da disciplina para o programa de formação do médico, é comum afirmações tais como:

[A disciplina é] Importante, pois nos dá uma boa visão do funcionamento do SUS e da gestão do cuidado. (Aluno turma 3)

Essa disciplina é muito pertinente e serve como um aprendizado de como aplicar o conteúdo aprendido em outras disciplinas na vida real. (Aluno turma 1)

Sobre a utilização do projeto terapêutico singular como eixo estruturante do curso, os alunos têm elaborado comentários favoráveis a esta proposta pedagógica, com afirmações como:

Pudemos elaborar um projeto terapêutico, o que fez com que pudéssemos compreender o funcionamento do sistema de saúde na prática. (Aluno turma 16)

A elaboração destes projetos tem estimulado os alunos a realizarem investigações em distintas instâncias da rede de saúde, o que vem possibilitando a vivência de cenários pedagógicos fora do centro de saúde. Ao buscarem contribuir para a qualificação da gestão do serviço e do cuidado ao usuário, os alunos vêm tendo a oportunidade de intercambiar experiências com os profissionais da rede, realizar visitas domiciliares e conhecer serviços de nível secundário do município. Esta experiência é registrada em falas, em geral elogiosas, quando comentam:

Foi importante o contato com vários gestores diferentes do Centro de Referência de Doenças Sexualmente Transmissíveis e AIDS (CRDSTAIDS), do Centro de Saúde, do Complexo Ouro Verde para ter diferentes visões. (Aluno turma 7)

Indagados sobre a relação com os trabalhadores de distintas unidades, afirmam:

Foram muito acolhedores (tanto no centro de saúde, quanto no centro de atenção psicossocial (CAPS) e no Tear das Artes). Em momento nenhum nos viram como intrusos. Sempre nos inseriram nas suas atividades. (Aluno turma 5) 
O contato também foi enriquecedor, pois observamos na prática a atuação: trabalho, problemas e resoluções destes, os quais dificilmente entraríamos em contato durante a graduação nas outras disciplinas. (Aluno turma 8)

É importante assinalar que o caminho percorrido pelas distintas turmas são diferentes uma vez que obedecem a uma combinação de fatores, entre os quais citamos, por sua relevância: o problema (demanda) proposto pelas equipes locais aos alunos, as estratégias de investigação/intervenção dos alunos e a viabilidade de implementação destas últimas.

Como muitas das estratégias são definidas a partir do contato dos alunos com demanda apresentada pelas equipes, faz-se necessário negociar, com o paciente, a família, os trabalhadores, os equipamentos sociais e os serviços secundários e terciários, visitas no curto espaço de dez dias. O maior ou menor sucesso desse processo depende da capacidade docente de produzir e sustentar compromissos com os vários atores por um lado e, por outro, articular conversas possibilitando uma rede de apoio junto aos gestores e aos trabalhadores dos serviços de saúde envolvidos. Esta articulação tem sido, na prática, um dos grandes desafios docentes, demandando capacidade específica e tempo "extraclasse". A receptividade da rede SUS/Campinas à proposta e a colaboração da direção distrital ao processo constituem elemento de maior importância para a "produção/construção" dos objetivos pedagógicos, o que referenda relatos de outros investigadores (Marins, 2004; Feuerwerker, Costa, Rangel, 2000).

A vivência dos alunos em serviços de atenção secundária do município, na disciplina SC/GP, constitui uma das poucas oportunidades para que o aluno de medicina conheça a complexidade e riqueza dos equipamentos do município, já que, no currículo atual, o ensino se realiza, na sua quase totalidade, nas unidades hospitalares da Unicamp e nas unidades básicas do município.

As diferenças e, em muitos casos, a complementaridade entre o nível secundário do município e a universidade, justificam, em nossa opinião, um esforço que objetive ampliar a inserção do aluno na rede municipal para além da rede básica. A vivência nos serviços secundários - CAPS, Núcleo de Apoio à Dependência Química, Serviço de Atenção e Internamento Domiciliar, Centro de Referência em Reabilitação, CRDSTAIDS e unidades de urgência/emergência, entre outros - constitui uma oportunidade única para o graduando ampliar o seu olhar sobre a rede de serviços e para reforçar a percepção da importância da rede de serviços para o sucesso de práticas clínicas e de cuidado.

É importante observar que a exiguidade da carga horária da disciplina determinou que se priorizasse, como cenário de práticas, a rede básica e secundária. Em alguns momentos, os alunos são estimulados a discutir problemas específicos em hospitais da rede SUS/Campinas que mantenham relação de referência com os serviços ou com os problemas de saúde/cuidado dos usuários acompanhados na disciplina.

Esta decisão, de caráter pragmático, não deve ser confundida com a impossibilidade, e mesmo com a necessidade, de se armar uma grade curricular objetivando a introdução de conteúdos de gestão do cuidado/atenção junto às práticas hospitalares. No caso da FCM/Unicamp, isto constituiria uma excelente oportunidade de reflexão para os alunos, uma vez que, durante o internato, estes passam grande parte de seus estágios em unidades hospitalares, no caso, no Hospital de Clínicas da Unicamp e no Hospital Regional Sumaré. O ensino de gestão durante os estágios clínicos dos últimos anos do ensino de graduação constituiria, no nosso entender, um aporte importante para os alunos e, ao mesmo tempo, um desafio para o ensino da área de Saúde Coletiva que, ainda hoje, encontra dificuldade para construir formulações e para definir sua inserção efetiva junto às unidades de atenção terciária.

\section{Sobre a parceria com os trabalhadores}

Nesses múltiplos cenários os alunos convivem com distintos profissionais. Ao serem perguntados sobre como avaliavam o contato com os trabalhadores das unidades, os alunos fizeram uma avaliação positiva do mesmo afirmando que:

[...] Interessante, principalmente quando foi relacionado ao caso do Sr. G. Pudemos conhecer a realidade desses trabalhadores, alguns problemas e dificuldades por eles enfrentados; a necessidade de um trabalho multiprofissional e interdisciplinar. (Aluno turma 3) 
REFLEXÕES SOBRE O ENSINO DE GESTÃO EM SAÚDE ...

O contato com os trabalhadores de saúde foi proveitoso e nos ensinou muito sobre a dinâmica do local. (Aluno turma 9)

Houve uma troca de conhecimentos. Produtivo para ambas as partes. (Aluno turma 12)

Observa-se aqui um intercâmbio bastante profícuo entre alunos e trabalhadores. Este intercâmbio, facilitado pela parceria com a rede e pela lógica tutorial de ensino do corpo docente (Venturelli, 2000), permitiu aos alunos captarem as diversas visões, por parte dos profissionais, sobre o trabalho em saúde e o conhecimento aprofundado do cotidiano dos serviços de saúde.

Como já relatado, os docentes vêm buscando contribuir para o processo de educação permanente dos trabalhadores. Isto não tem impedido que, em alguns momentos, esta relação ocorra de maneira conflituosa ou limitada. Alguns dos elementos que demandam do corpo docente uma postura atenta e interativa são: a ocorrência de conflitos éticos, críticas ásperas do corpo discente em relação ao serviço, o sentimento de invasão de privacidade por parte dos trabalhadores, e a não disponibilidade de parte dos funcionários para atender as necessidades da disciplina. Constituem, igualmente, uma oportunidade para se discutirem vivências cotidianas em torno do trabalho em saúde e suas repercussões na qualidade da atenção prestada.

\section{Sobre o processo de avaliação dos alunos}

$\mathrm{Na}$ avaliação dos alunos, temos valorizado o componente formativo, e, nelas, os itens: assiduidade, postura ética, compromisso com os usuários, participação e capacidade reflexiva.

Ao serem perguntados sobre a adequação dos mencionados critérios de avaliação, os alunos deram nota 8,98 (média das notas globais atribuídas à disciplina pelos 64 alunos que responderam aos questionários de avaliação da disciplina), comentando que:

Desde o início foram pactuados os objetivos e os meios de chegar até eles. [...] O conteúdo ministrado era sempre discutido, e de certa maneira, já estávamos sendo avaliados (o professor sempre fez questão de discutir as impressões de cada um, o que "tinha ficado" sedimentado de determinado assunto. (Aluno turma 4)

A avaliação está fundada na participação, envolvimento e na apresentação de trabalhos dos alunos. Julgo que é uma forma completa de avaliação e o principal não se funda em modelos desgastados e automáticos de devolutiva do conhecimento. (Aluno turma 8)

Valorizou a experiência prática, além de estar aberto à discussão de como seriam as melhores formas e critérios de avaliação. (Aluno turma 12)

Entre os pontos levantados de forma crítica pelos alunos, vale destacar a existência de uma desigualdade entre os critérios utilizados pelos professores na avaliação dos vários grupos, sobretudo quanto à nota sobre postura. Criticaram, algumas vezes, a obrigatoriedade de textos considerados de difícil leitura e, em alguns casos, a quantidade de leitura indicada. Questionaram, também, a impropriedade de que professores com inserção vertical e excepcional (p.ex. quatro horas-curso) pudessem avaliar alunos.

\section{Sobre a rede docente}

Esta última crítica reafirma nossa opinião sobre a pertinência e a importância de criação de vínculos estáveis e longitudinais entre o docente e o grupo de alunos. Na prática, isto tem constituído um grande desafio organizativo, uma vez que, na tradição de ensino de graduação na FCM/Unicamp, é difícil conseguir um professor com disponibilidade de carga horária nos dez dias consecutivos.

Procuramos superar este entrave por meio da participação horizontal de um docente/referência com, no 
mínimo, seis períodos/turma. A composição de dupla de professores por turma tem sido igualmente importante, já que não somente facilita o vínculo como permite o intercâmbio constante de reflexões e de relatos entre os docentes responsáveis.

Importante mencionar que o corpo docente encarregado da disciplina contou, no período investigado, com apenas dois professores do quadro permanente da FCM/Unicamp. Note-se que há atividades durante cinco períodos por semana, durante 12 meses consecutivos, perfazendo $960 \mathrm{hs} /$ ano de atividades presenciais. No internato, há interrupção somente durante os 15 dias de férias coletivas da Unicamp, nos feriados de Natal e de Ano Novo.

Tem-se buscado lidar com estas limitações por intermédio da inclusão, nas atividades de ensino, de professores/pós-graduandos vinculados aos professores regulares. Busca-se, sempre que possível, incluir os pós-graduandos no Programa de Estágio Docente, mecanismo institucional da Universidade de Campinas que tem como objetivo aprimorar a capacidade docente dos alunos de pós-graduação (Unicamp, 2007). Os critérios na escolha dos referidos estagiários são: domínio teórico dos conteúdos, competência pedagógica para atividades tutoriais e, elemento importantíssimo, experiência profissional junto a serviços públicos de saúde.

Explorando e tensionando as fronteiras do encontro academia/serviços, tem-se logrado, a exemplo de outras experiências, constituir uma "rede de apoio docente" formada por trabalhadores dos serviços visitados e que vêm assumindo responsabilidades crescentes junto ao corpo discente da disciplina (Carvalho, Garcia, Rocha, 2006). A importância desta rede é reconhecida por muitos alunos quando afirmam que

O contato foi intenso, uma experiência enriquecedora pois tivemos contato com agentes de saúde, de enfermagem, enfermeiros, médicos, diretores de centros de saúde. Com isso observamos o trabalho de cada membro da equipe o que nos permitiu ter acesso a todos os níveis de atuação dos trabalhadores de saúde. (Aluno turma 8)

Os profissionais se mostraram abertos tanto para nos ensinar coisas quanto para ouvirem críticas. Sempre se mostraram muito atenciosos. (Aluno turma 4)

Fomos muito bem "acolhidos" pelos diversos profissionais, sempre dispostos a nos responder questões que tínhamos e nos falar de suas dificuldades, idéias, projetos... Muito proveitoso. (Aluno turma 5)

Tem sido central na estruturação deste apoio docente o papel dos gestores estimulando a parceria e, indo além, valorizando esta última como um dispositivo de qualificação do trabalho nas unidades de saúde. Os alunos reconhecem a importância dos coordenadores da unidade nos seguintes comentários:

A gestora mostrou-se muito disposta com os alunos, mobilizando a equipe para as discussões. Sem isso o módulo não teria funcionado. (Aluno turma 6)

Nos deixaram à vontade para participar das discussões. Se puseram à disposição para conversar e tirar dúvidas. Ajudaram na organização de como os outros funcionários nos ajudariam. (Aluno turma 5)

A diretora nos recebeu muito bem, sempre preocupada em nos mostrar o que faziam os gestores e também as dificuldades presentes nesse posto de trabalho. (Aluno turma 4)

As afirmativas reforçam o papel central que os gestores locais têm tido para o bom funcionamento da disciplina, uma vez que articulam a relação entre alunos e os trabalhadores, bem como asseguram a operacionalização da disciplina, incorporando o estágio como um dispositivo no cotidiano gerencial do serviço, disparando processos de educação permanente na unidade e apoiando, como um quase-tutor, o trabalho realizado com os alunos. 
REFLEXÕES SOBRE O ENSINO DE GESTÃO EM SAÚDE ...

\title{
O internato em gestão como analisador do ensino de Saúde Coletiva
}

O processo sistemático de avaliação da disciplina pelos alunos graduandos tem produzido questionamentos sobre a experiência que estes alunos, já cursando o $5^{\circ}$ ano, tiveram ao longo de sua formação com várias disciplinas ligadas ao DMPS. Isto se manifestou nas seguintes ponderações:

\begin{abstract}
Ela é muito pertinente, no entanto merece ser melhor distribuída no conteúdo dos demais anos - acredito que já esteja ocorrendo - para ser melhor aproveitada. (Aluno turma 8)

Acredito que o tema é fundamental, mas seria válido se ele fosse ministrado da forma como foi agora [no $5^{\circ}$ ano de graduação] já antes de atendermos por 1 ano nos centros de saúde [4 $4^{\circ}$ ano], pois acredito que nossa passagem pelos centros de saúde no $4^{\circ}$ ano teria sido melhor aproveitada - poderíamos ter oferecido uma atenção mais integral. (Aluno turma 5)
\end{abstract}

Estas impressões vêm sendo debatidas e aprofundadas no interior do grupo gestor da disciplina, com os alunos e com a Comissão de Ensino da FCM/Unicamp. Estas falas revelam indagações que nos parecem centrais ao processo institucional de reforma curricular em curso. Elas dizem respeito ao programa teórico, à proposta pedagógica da Saúde Coletiva e àquelas atividades vinculadas ao ensino das distintas especialidades clínicas, e podem ser sintetizadas em duas questões: a) o ensino de Saúde Coletiva na graduação da FCM/Unicamp, e em outras Universidades, apresenta coerência doutrinária, metodológica e de conteúdo ao longo dos diferentes anos e disciplinas? b) está ocorrendo de fato uma integração entre os conteúdos da Saúde Coletiva e os ministrados pelas áreas clínicas? Em nossa opinião, as intrínsecas relações entre gestão e clínica demandam a superação do pensamento dual vigente em nossas instituições de ensino, que fragmenta e separa o que na vida real constituem interfaces de um mesmo movimento. Coerentes com esta afirmação, julgamos que os temas trabalhados no internato podem (e devem) ser ministrados ao longo dos seis anos do curso de medicina, tanto nas disciplinas do DMPS quanto nas ministradas pelos demais departamentos e, em especial, naqueles voltados para o ensino da clínica.

\section{Considerações finais}

Entendemos que o recorte temático do internato - gestão da clínica/gestão do cuidado/gestão em rede - potencializa a parceria que se realiza no cotidiano da disciplina, facilitando a relação entre docentes, alunos, trabalhadores e gestores. A experiência aqui relatada parece indicar a pertinência de que as referidas temáticas venham a constituir um dos eixos do currículo de graduação de médicos e das profissões em saúde na área de Políticas, Gestão e Planejamento. Estes saberes devem, igualmente, configurar uma ferramenta conceitual que possibilite o acercamento entre a clínica e a gestão do trabalho em saúde nos macro e microespaços do cotidiano.

Destacamos, também, o empenho dos gestores locais e o compromisso ético de muitos trabalhadores como fatores essenciais para se concretizar a parceria entre as diferentes instituições participantes. O compartilhamento da gestão do processo de ensino aprendizagem, assim como a instituição de espaços coletivos e sistemáticos de debates e de avaliação, têm sido centrais ao processo.

Ao valorizar as demandas das equipes e o trabalho conjunto, a disciplina SC/GP se orienta por uma singular incorporação da metodologia fundada na discussão de casos que tem procurado estimular a maioria dos alunos a ter um papel ativo no processo de investigação e na elaboração de projetos terapêuticos singulares envolvendo indivíduos e coletivos.

Ao buscar contribuir para uma formação de competências na gestão da atenção em saúde para os alunos, a SC/GP tem levado em conta a discussão de problemas reais que são apontados pelos trabalhadores das distintas unidades. Esta dinâmica, à qual acrescentamos a parceria realizada com trabalhadores e gestores durante o estágio, tem contribuído para o aprimoramento da formação dos trabalhadores e para que os serviços possam refletir sobre a gestão que neles se realiza. Ocorre aqui um 
encontro entre o aprendizado significativo, por parte dos alunos, com processos de educação permanente, que explica, em boa parte, a avaliação positiva do processo.

Concluímos este trabalho afirmando a necessidade de novos estudos que aprofundem o que aqui foi relatado e que busquem captar distintos aspectos desta instigante experiência, e, em especial, averiguar a sustentabilidade do referido projeto a médio e longo prazo.

\section{Colaboradores}

Os autores participaram igualmente da formulação, redação e revisões do manuscrito.

\section{Referências}

BERBEL, N.A.N. Metodologia da problematização: uma alternativa metodológica apropriada para o ensino superior. Semina, v.17, n. esp., p.9-19, 1995.

CAMPINAS. Projeto Paidéia de saúde da família. Campinas, 2001. (mimeogr.)

CAMPOS, G.W.S. Um método para análise e co-gestão de coletivos. São Paulo: Hucitec, 2000.

Reforma da reforma: repensando a Saúde. São Paulo: Hucitec, 1992.

CARVALHO, S.R. Saúde Coletiva e promoção da saúde: sujeito e mudança. São Paulo: Hucitec, 2005.

CARVALHO, S.R.; CAMPOS, G.W.S. Modelos de atenção à saúde: a organização de equipes de referência na rede básica da Secretaria Municipal de Saúde de Betim/Minas Gerais. Cad. Saude Publica, v.6, n.2, p.507-15, 2000.

CARVALHO, S.R.; CUNHA, G.T. A gestão da atenção na saúde: elementos para se pensar a mudança da organização na saúde. In: CAMPOS, G.W.S. et al. (Orgs.). Tratado de Saúde Coletiva. São Paulo: Hucitec, 2006. p.837-68.

CARVALHO, S.R.; GARCIA, R.A.; ROCHA, D.C. O ensino da Saúde Coletiva no curso médico da Unicamp: experiências inovadoras junto a unidades básicas de saúde. Interface - Comunic., Saude, Educ., v.10, n.20, p.457-72, 2006.

CECílIO, L.C.O. As necessidades de saúde como conceito estruturante na luta pela integralidade e eqüidade na atenção em saúde. In: PINHEIRO R.; MATTOS, R.A. (Orgs.). Os sentidos da integralidade. Rio de Janeiro: IMS/ABRASCO, 2001. p.39-64.

CUNHA, G.T. A clínica ampliada na atenção básica. São Paulo: Hucitec, 2005.

FEUERWERKER, L.C.M.; COSTA, H.; RANGEL, M.L. Diversificação de cenários de ensino e trabalho sobre as necessidades/problemas da comunidade. Divulg. Saude Debate, v.22, p.36-48, 2000.

LIMA, V.V. Avaliação de competência nos cursos médicos. In: MARINS, J.J.N. et al. (Orgs.). Educação médica em transformação: instrumentos para a construção de novas realidades. São Paulo: Hucitec, ABEM, 2004. p.123-42. 
REFLEXÕES SOBRE O ENSINO DE GESTÃO EM SAÚDE ...

MARINS, J.J.N. Os cenários de aprendizagem e o processo de cuidado em saúde. In: et al. (Orgs.). Educação médica em transformação: instrumentos para a construção de novas realidades. São Paulo: Hucitec, ABEM, 2004. p.97-108.

MERHY, E.E. Saúde: a cartografia do trabalho vivo. São Paulo: Hucitec, 2002.

A perda da dimensão cuidadora na produção da saúde. In: CAMPOS, C.R. et al. (Orgs.). Sistema Único de Saúde em Belo Horizonte: reescrevendo o público. São Paulo: Xamã, 1998. p.103-20.

OLIVEIRA, G.N. O projeto terapêutico como contribuição para a mudança das práticas de saúde. 2007. Dissertação (Mestrado) - Faculdade de Ciências Médicas, Universidade Estadual de Campinas, Campinas. 2007.

SILVA JR., A.G. Modelos tecnoassistenciais em saúde: o debate no campo da Saúde Coletiva. São Paulo: Hucitec, 1998.

UNICAMP. Programa de estágio docente, 2007. Disponível em: <http:// www.prpg.unicamp.br/bolsas_estagiodocente.phtml>. Acesso em: 20 jun. 2007.

VENTURELLI, J. Educación médica: nuevos enfoques, metas y métodos. Salud Soc., v.5, p.307, 2000. 
Este artigo descreve e analisa o internato em gestão e planejamento em saúde do quinto ano de medicina da Faculdade de Ciências Médicas da Universidade de Campinas. Foram utilizados, como materiais para análise: documentos oficiais, resultados de questionário semiestruturado aplicado aos alunos para avaliarem a disciplina, relatórios provenientes de reuniões e monografias produzidas pelos alunos durante o primeiro ano de funcionamento do curso, a partir de novembro de 2004. Optou-se por recortar o campo da gestão, enfocando-se centralmente a gestão do trabalho em saúde. A estratégia pedagógica empregada no curso baseou-se, sobretudo, na discussão de caso e elaboração de projetos terapêuticos e de intervenção. A avaliação dos alunos tanto validou a experiência e a estratégia pedagógica, quanto apresentou problemas e sugestões de mudança.

Palavras-chave: Educação em saúde. Ensino médico. Planejamento e gestão. Reforma curricular. Atenção à saúde. Administração em Saúde.

Reflections on health management teaching in the medicine internship of the School of Medical Sciences - State University of Campinas - Unicamp

This article describes and analyzes the Health Planning and Management internship for undergraduate students who are in the fifth year of the School of Medical Sciences of the State University of Campinas (UNICAMP). We analyzed official documents, results from a semi-structured questionnaire administered to the students, in which they evaluated the discipline, reports from meetings, and monographs produced by the students during the course's first year, from November 2004 onwards. We decided to focus, within the Health Planning and Management field, on the investigation of the work management process. The pedagogical strategy employed in the course was based, mainly, on clinical case studies and design of therapeutic and intervention projects. The students' evaluation validated the experience and the pedagogical strategy, and also discussed problems and suggested changes.

Keywords: Health education. Medical education. Planning and management. Curricular reform. Health care. Health administration.

Reflexiones sobre la enseñanza de gestión en salud en el internato de medicina en la Facultad de Ciencias Médicas de la Universidad Estatal de Campinas, Unicamp, estado de São Paulo, Brasil

Este artículo describe y analiza el internato en gestión y planeamiento en salud del quinto año de medicina de la Facultad de Ciencias Médicas de la Universidad de Campinas. Se utilizaron como materiales para análisis documentos oficiales, resultados de cuestionario semi-estructurado aplicado a los alumnos para que valoren la disciplina, informes provenientes de reuniones y monografías producidas por los alumnos durante el primer año de funcionamiento del curso, a partir de noviembre de 2004. Se optó por recortar el campo de gestión, enfocándose centralmente la gestión del trabajo en salud. La estrategia pedagógica empleada en el curso se basó principalmente en la discusión del caso y elaboración de proyectos terapéuticos y de intervención. La valoración de los alumnos validó tanto la experiencia y la estrategia pedagógica como presentó problemas y sugerencias de cambio.

Palabras clave: Educación en salud. Enseñanza médica. Planeamiento y gestión. Reforma curricular. Atención a la salud. Administración en salud. 